\title{
Determination of 3D Trajectories of Knots in Solar Prominences Using MSDP Data
}

\author{
Maciej Zapiór · Pawel Rudawy
}

Received: 30 June 2010 / Accepted: 19 September 2010 / Published online: 23 October 2010

(C) The Author(s) 2010. This article is published with open access at Springerlink.com

\begin{abstract}
In this paper we present a new method of restoration of the true three-dimensional (3D) trajectories of prominence knots using ground-based observations taken with a single telescope that is equipped with a Multi-Channel Subtractive Double Pass imaging spectrograph. Our method allows us to evaluate the true 3D trajectories of the prominence knots without any assumptions concerning the shape of the trajectories or the dynamics of the motion. The reconstructed trajectories of several knots observed in three prominences are presented.
\end{abstract}

Keywords Instrumentation and data management · Prominences, dynamics · Prominences, formation and evolution

\section{Introduction}

All solar prominences show motions of various spatial and temporal scales, ranging from small-scale and slow displacements of individual structures (knots) in quiet prominences to violent eruptions of the whole structure in eruptive prominences. Investigation of the true three-dimensional (3D) trajectories of the individual knots of the evolving prominences can provide very valuable data concerning the spatial structure and dynamics of the prominences themselves and their magnetic skeletons, the overall structure of huge magnetic systems encompassing prominences, as well as coronal mass ejection-prominence relations. The observations and investigations of the magnetic fields in solar prominences are very difficult due to insufficient spatial resolution and low sensitivity of direct or indirect measurement

Electronic supplementary material The online version of this article (doi:10.1007/s11207-010-9643-z) contains supplementary material, which is available to authorized users.

M. Zapiór $(\bowtie) \cdot$ P. Rudawy

Astronomical Institute of the University of Wrocław, ul. Kopernika 11, 51-622 Wrocław, Poland

e-mail: zapior@astro.uni.wroc.pl

P. Rudawy

e-mail: rudawy@astro.uni.wroc.pl 
methods of the magnetic fields currently used. For this reason the observations of the bulk flows along the magnetic ropes provide us with very valuable data.

Until now most data concerning trajectories and kinematics of prominence material has been based on analysis of filtergram observations (e.g., images taken in various spectral lines, mostly in the $\mathrm{H} \alpha$ line) and spectral observations taken with classical spectrographs. The analysis of time series of images provides parameters of the motion of the observed structure (its translation, velocity, and acceleration) only in a plane of the sky (e.g., Rothschild, Pecker, and Roberts, 1955; McCabe and Fisher, 1970; Rompolt, 1975; Kim, 1990; Aurass et al., 1991; Vršnak et al., 1993; Uddin, Gaur, and Pande, 1994; Tandberg-Hanssen, 1995; Rudawy and Madjarska, 1998), while 3D trajectories of the plasma (separated knots of material) could be evaluated only under certain strong and arbitrary assumptions. To do this, Billing and Pecker assumed that the acceleration of the prominence knots is always normal relative to their velocity vector (Billings and Pecker, 1954), Paluš assumed spiral motions of the knots on conical surfaces (Paluš, 1972), Makhmudov and coauthors assumed constant acceleration of the falling knots (Makhmudov, Nikolsky, and Zhugzda, 1980), and Ballester and Kleczek introduced their own method based on an extended geometrical analysis of the data (Ballester and Kleczek, 1983). For data taken with classical spectrographs, which have a very limited field of view and/or time resolution, it is possible to evaluate line-of-sight (LOS) velocities as well as transverse translations of the observed separated structure (using slit-jaw images), but relative slowness of the scanning process of the entrance slit across the observed prominence substantially reduces the temporal resolution of the observations. Due to the described limitations, only a small volume of data collected with ground-based instruments related to the $3 \mathrm{D}$ trajectories and kinematics of the prominence plasma is available presently.

The 3D spatial structure and evolution of prominences or coronal mass ejections (CMEs) could be investigated using observations collected simultaneously with two distant instruments (like Solar Terrestrial Relations Observatory's A and B twin satellites), sufficiently separated to restore individual locations in 3D space by means of trigonometric parallaxes. Very valuable data concerning 3D evolution of the CMEs have already been collected with these satellites, but the amount of such data evaluated for prominences is unfortunately lower (Panasenco and Martin, 2007; Slater and Aschwanden, 2007; Bemporad, 2009; Liewer et al., 2009).

Due to the unique optical characteristics of Multi-Channel Subtractive Double Pass (MSDP) imaging spectrographs (large field of view, possible short exposure times, and high temporal cadences in scanning mode), collected data are convolutions of the 2D images and spectra (Mein, 1991a, 1991b; Rompolt et al., 1993). The method discussed in this work is suitable for analysis of the spatial motions and evolution of whole extended structures like big eruptive prominences visible over the solar limb or filaments on the solar disk. The method restores the true 3D trajectories of the prominence knots based on ground-based observations taken with a single telescope equipped with an MSDP imaging spectrograph. Our method allows us to evaluate $3 \mathrm{D}$ trajectories of the prominence knots without any assumptions concerning the shape of the trajectories or the dynamics of the motion. The reconstructed trajectories of several blobs observed in three prominences are presented.

\section{Observational Material and Data Processing}

The observational data were collected using the Large Coronagraph of the University of Wrocław, equipped with the MSDP imaging spectrograph, installed in the Białków Observatory. The coronagraph has a $51 \mathrm{~cm}$ entrance aperture, and a nearly $14.5 \mathrm{~m}$ effective focal 
length, although its real spatial resolution is limited by seeing only to about 1 arcsec. The MSDP spectrograph has a nine-channel prism-box creating $0.4 \AA$ steps in wavelength between consecutive "channels" forming spectro-images and defining in this way a spectral resolution of the collected data (Rompolt et al., 1993). Consecutive channels cover exactly the same area on the Sun $\left(325 \times 41 \operatorname{arcsec}^{2}\right)$, but each of these is recorded in a slightly shifted waveband in relation to others.

Three series of prominence observations were selected from archive data covering the period 1996-2006 and data collected from August-September 2009.

The numerical reduction of the raw spectro-images was made using a standard MSDP software designed by Mein (1991b) and modified by Rudawy (1996) and our own auxiliary codes. After numerical processing for each raw spectro-image, we obtained a compound file containing 13 images in different wavelengths in the neighborhood of the $\mathrm{H} \alpha$ line $(\Delta \lambda=0$, $\pm 200 \mathrm{~m} \AA, \pm 400 \mathrm{~m} \AA, \pm 600 \mathrm{~m} \AA, \pm 800 \mathrm{~m} \AA, \pm 1000 \mathrm{~m} \AA, \pm 1200 \mathrm{~m} \AA$ ) and a narrow field of view (defined by the entrance window of the spectrograph). Next, after numerical "sticking" of these images for each scan we got a compound file with 13 large-area images covering the investigated prominence. Usually we added a dozen or so images to receive a single large-area image, and we made several tens of scans for each observed prominence, reaching a temporal resolution of the order of $1 \mathrm{~min}$.

Due to the rotation of the field of view (the observations were made in coudé focus of the Large Coronagraph) and instabilities in the pointing of the telescope, we de-rotated and translated numerically all images of the investigated prominence to the common reference frame of an arbitrary orientation and starting point. As reference structures we used sunspots, some stable structures on the limb (the place where they cross the solar limb), or stable feet of the prominences. Axes of the reference frames were arbitrarily oriented as follows: $x$ axis, from left to right, $y$ axis, from top to bottom (both in the sky plane), $z$ axis, oriented from the observer to the Sun. The starting positions of the knots along the $z$ axis were not known; they were always assumed to be equal to zero.

Well-defined knots of prominence material visible on at least five consecutive images were selected for further detailed analysis. An $x-y$ position of the blob was estimated on the consecutive images using the position of the centroid of the knot's emission (see Figure 1) delimited by an arbitrary selected isophote (usually the isophote of $70 \%$ of the brightest knot's emission). The newest version of our software automatically points out knot positions on consecutive images, but results are examined by the observer to prevent bad tracking. In an automatic mode the isophotes in the range of $70-90 \%$ were automatically fitted to delimit the analyzed knot from other structures. The mean $\mathrm{H} \alpha$ emission profiles of the knots were used to evaluate LOS velocities of the material. As a result, for each investigated knot we obtained a time series of its positions in the plane of the sky (expressed in kilometers) and its LOS velocities (in kilometers per second) at different times $\left(t_{i}\right)$.

The temporal changes of the knot's spatial position and spatial velocity were calculated using an approximation of the obtained data $x\left(t_{i}\right), y\left(t_{i}\right)$, and $V_{\mathrm{LOS}}\left(t_{i}\right)$ with polynomials $\left(x_{\text {poly }}(t), y_{\text {poly }}(t), V_{\text {LOS, poly }}(t)\right)$ and then by integration:

$$
z_{\text {poly }}(t)=\int_{0}^{t} V_{\mathrm{LOS}, \text { poly }}\left(t^{\prime}\right) \mathrm{d} t^{\prime}
$$

in order to obtain the translation along the $z$ axis. Calculations were alternatively made by interpolation of the smoothed data $\left(x_{\text {smooth }}\left(t_{i}\right), y_{\text {smooth }}\left(t_{i}\right), V_{\mathrm{LOS} \text {,smooth }}\left(t_{i}\right)\right)$ with spline functions $\left(x_{\text {spline }}(t), y_{\text {spline }}(t), V_{\mathrm{LOS} \text {,spline }}(t)\right)$ and again by integration:

$$
z_{\text {spline }}(t)=\int_{0}^{t} V_{\text {LOS }, \text { spline }}\left(t^{\prime}\right) \mathrm{d} t^{\prime} .
$$



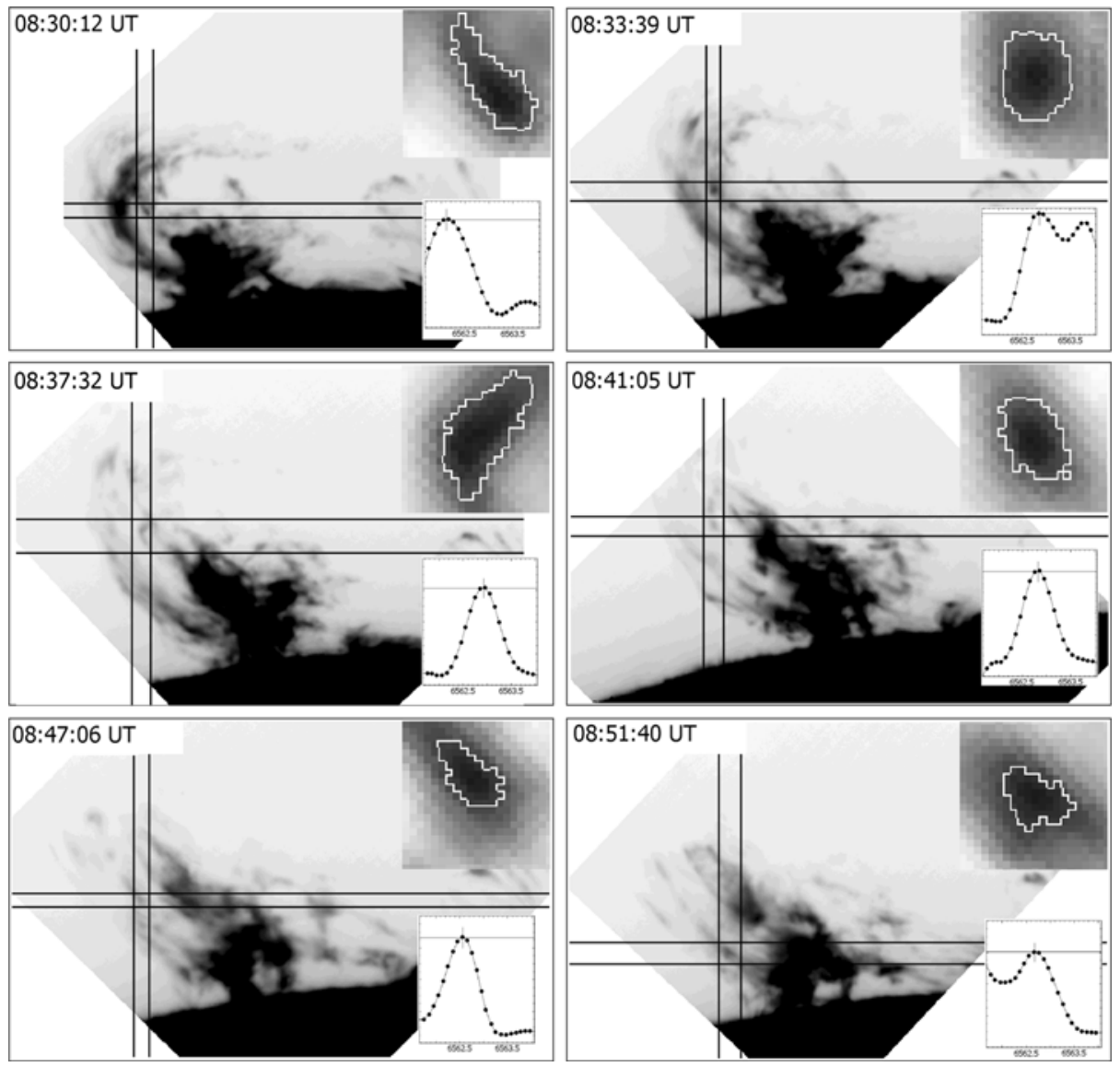

Figure 1 Images of an eruptive prominence observed on June 1, 2003 near active region NOAA 10375. The images were taken with the MSDP imaging spectrograph in the center of the $\mathrm{H} \alpha$ line. The double-line cross surrounds the analyzed knot at its center. Zoomed images (top right) and mean H $\alpha$ profiles (bottom right corner) of the knot are presented in each panel.

The degrees of the polynomials used for the approximation were chosen for $x\left(t_{i}\right), y\left(t_{i}\right)$, and $V_{\mathrm{LOS}}\left(t_{i}\right)$ independently. In case of interpolation we smoothed raw data by the running mean to eliminate the influence of bad data. The time series of 3D coordinates $(x(t), y(t), z(t))_{\text {poly }}$ or $(x(t), y(t), z(t))_{\text {spline }}$ directly define tracks of the knots in space. Figure 2 presents two versions of the trajectory of the same knot calculated using a polynomial approximation and a spline-function interpolation. Usually the trajectories calculated using a polynomial approximation were smoother than the trajectories calculated using the spline-function interpolation. While shapes of the majority of the magnetic loops observed in the solar corona can be well described by low-order polynomials, we decided to use polynomial approximation for all trajectories presented in the next section of the paper.

As mentioned before, due to the obvious observational limitation we assumed arbitrarily for each $\operatorname{knot} z(t=0)=0$, where $t=0$ was the beginning of the knot's observation period. In both methods we also calculated velocities along the $x$ and $y$ axes ( $v_{x}$ and $v_{y}$ respectively) as first derivatives $x_{\text {poly }}(t)$ and $y_{\text {poly }}(t)$ (or $x_{\text {spline }}(t)$ and $\left.y_{\text {spline }}(t)\right)$ and full spatial velocities 

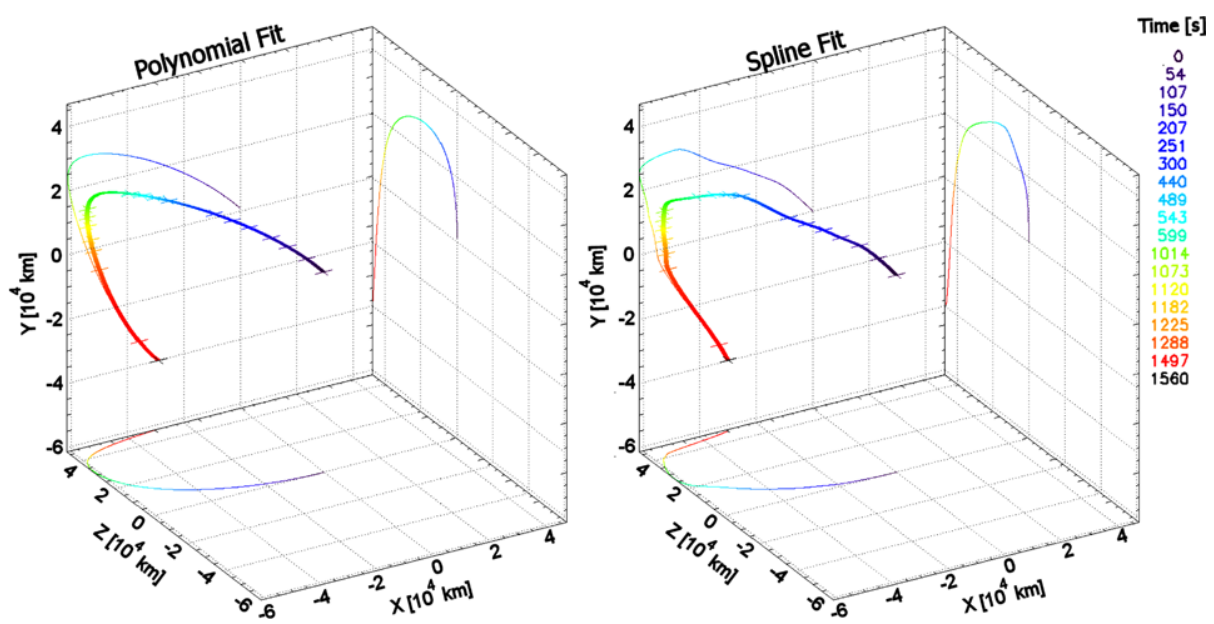

Figure 2 3D trajectory of knot $A$ observed in the eruptive prominence on June 1, 2003. Left and right panels: polynomial approximation and spline interpolation of the observational data, respectively. Projections of the restored 3D track on the $X-Z$ and $Y-Z$ planes (both are perpendicular to the plane of the sky) as well as on the plane of the sky $(X-Y)$ are plotted with thin lines. The time evolution is coded in color (violet - start, red - end of observations). Axes are scaled in kilometers.

$\left(v=\sqrt{v_{x}^{2}+v_{y}^{2}+v_{z}^{2}}\right)$ and accelerations along the path $\left(a=\frac{\mathrm{d} v}{\mathrm{~d} t}\right)$ (see Figure 3$)$. The obtained results are presented on Figures 5, 7, and 9.

Errors of the evaluated positions of the individual knots in the sky plane were estimated as the standard deviation of the positions of the reference structure in all analyzed images, from which we obtained its mean value (the main source of error was inaccuracies in coalignment of the images). The estimated errors in $x$ and $y$ are less than three pixels (approximately $1000 \mathrm{~km}$ on the Sun). Errors of Doppler velocity measurements $(\Delta V)$ were estimated for each knot separately using deviations of the individual measurements from their approximating polynomial. The error of the translation along the LOS was assumed to be equal to the product of $\Delta V$ and the number of temporal steps used in the integration of the velocity curve from the first observed position of the knot. Thus, the error of the $z$ coordinate accumulated along the integration (see Figure 3).

\section{Results}

\subsection{Post-Flare Loop System on July 14, 2005}

An extended post-flare loop system (PFLS) was observed from 9:22:54 to 9:46:03 UT on July 14, 2005, on the west solar limb over an active region NOAA 10786. The magnetic class of the region was $\beta \gamma$, and it produced several tens of $\mathrm{C}$ and $\mathrm{M}$ class solar flares, according to GOES (Geostationary Operational Environmental Satellite) classification. Five flares occurred shortly before the period of our observations: an M9.1 GOES-class flare at 05:57 UT and four C-class flares during the period lasting from 06:35 UT to 08:54 UT. The observed PFLS appeared after the M-class flare. The Large Angle and Spectrometric Coronagraph (LASCO) on board the Solar and Heliospheric Observatory (SOHO) satellite recorded a CME from the closest vicinity of the active region at 05:40 UT. 

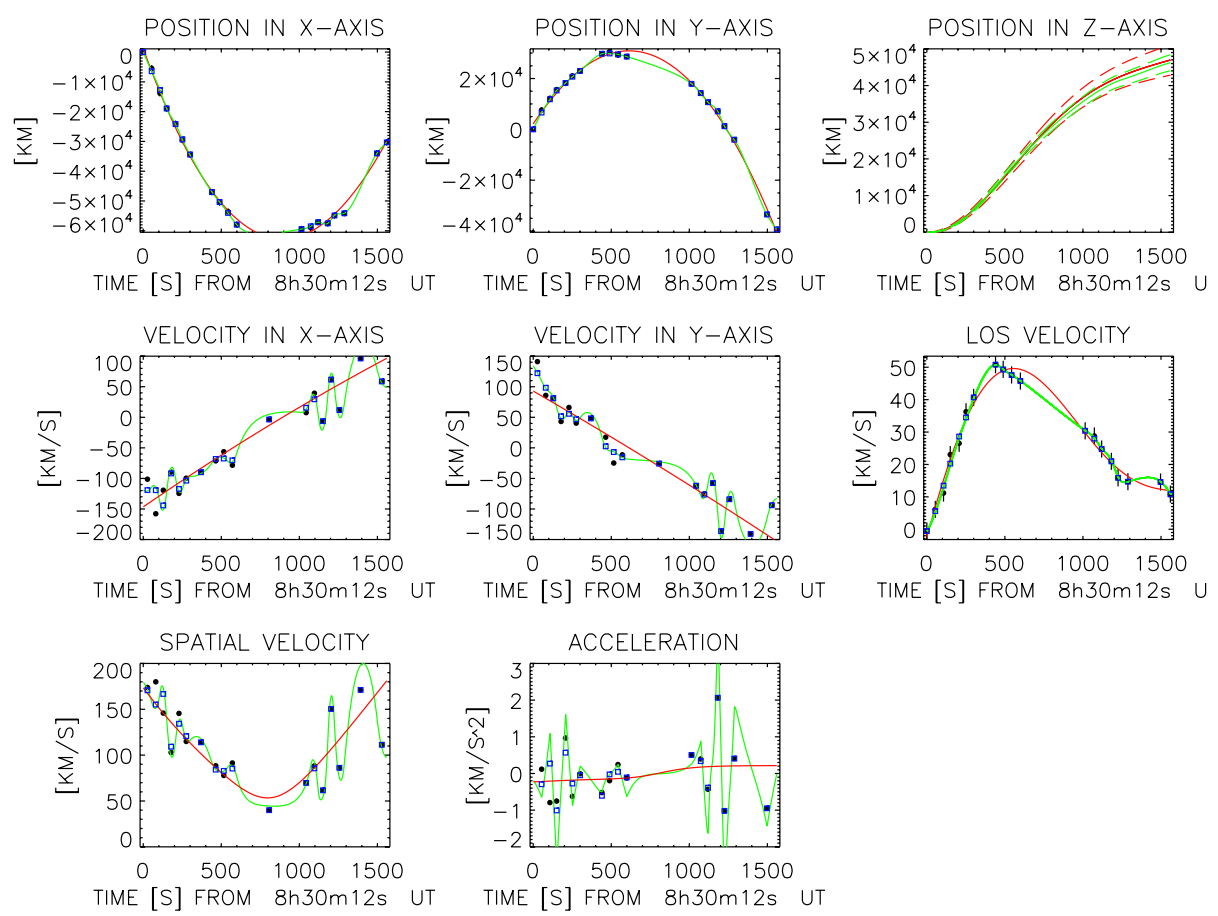

Figure 3 Kinetic properties of the trajectory of blob A observed in the eruptive prominence on June 1, 2003. Panels from top to bottom and from left to right: coordinates of the knot along $X, Y$, and $Z$ axes $(x$ and $y$ positions observed, $z$ position calculated), $v_{x}, v_{y}$, and $v_{z}$ velocities ( $v_{z}$ observed, $v_{x}$ and $v_{y}$ calculated), full spatial velocity and acceleration along the trajectory. Raw data are marked with black points, smoothed data are marked with squares, solid red and green lines indicate spline and polynomial approximation, respectively. Error bars are marked by vertical bars. In the top right panel the cumulative error of the integrated $z$ position is presented for the polynomial approximation (red dashed lines) and for spline interpolation (green dashed line).

The PFLS encompassed several loop-like structures and well-separated knots, from which we selected three knots (marked A, C, and D) for detailed analysis (see Table 1 for data details). Figure 4 shows trajectories of the knots projected onto the sky plane, while Figure 5 presents restored 3D tracks of the same knots. The axes of the reference system are scaled in kilometers, and the LOS axis is vertical. The tracks are presented in the 3D space and overlaid onto an image of the PFLS taken in the $\mathrm{H} \alpha$ line at 09:36:02 UT. Projections of the restored 3D tracks on the $X-Z$ and $Y-Z$ planes (both are perpendicular to the plane of the sky) as well as observed positions of the knots in the plane of the sky $(X-Y)$ are also shown. For clarity, $Z$ coordinates of starting positions of all knots are arbitrarily shifted in such a way that the most distant position on each trajectory lies in the plane of the sky $(X-Y)$. An animated version of Figure 5 is available in the electronic version of the paper.

The knot marked D was located inside the PFLS from 09:22:54 to 09:39:10 UT on 23 consecutive images. At the beginning of the observational period it had a positive Doppler velocity $\left(35 \mathrm{~km} \mathrm{~s}^{-1}\right)$, but afterwards it decelerated gradually to zero and then accelerated to $40 \mathrm{~km} \mathrm{~s}^{-1}$. Thus, its 3D track is bent out from the observer (see Figure 5). Two other knots (marked A and C) were observed outside the PFLS (see Figures 4 and 5). 
Table 1 Knots observed in the post-flare loop system on July 14, 2005.

\begin{tabular}{llll}
\hline Knot & Start obs. & End obs. & No. of images \\
\hline A & $9: 24: 09$ & $9: 46: 03$ & 33 \\
C & $9: 34: 10$ & $9: 46: 03$ & 20 \\
D & $9: 22: 54$ & $9: 39: 10$ & 23 \\
\hline
\end{tabular}

Figure 4 Observed positions of three knots (marked A, C, and D) of the post-flare loop system observed on July 14, 2005. The image was taken with the MSDP imaging spectrograph in the center of the $\mathrm{H} \alpha$ line at 09:36:02 UT.
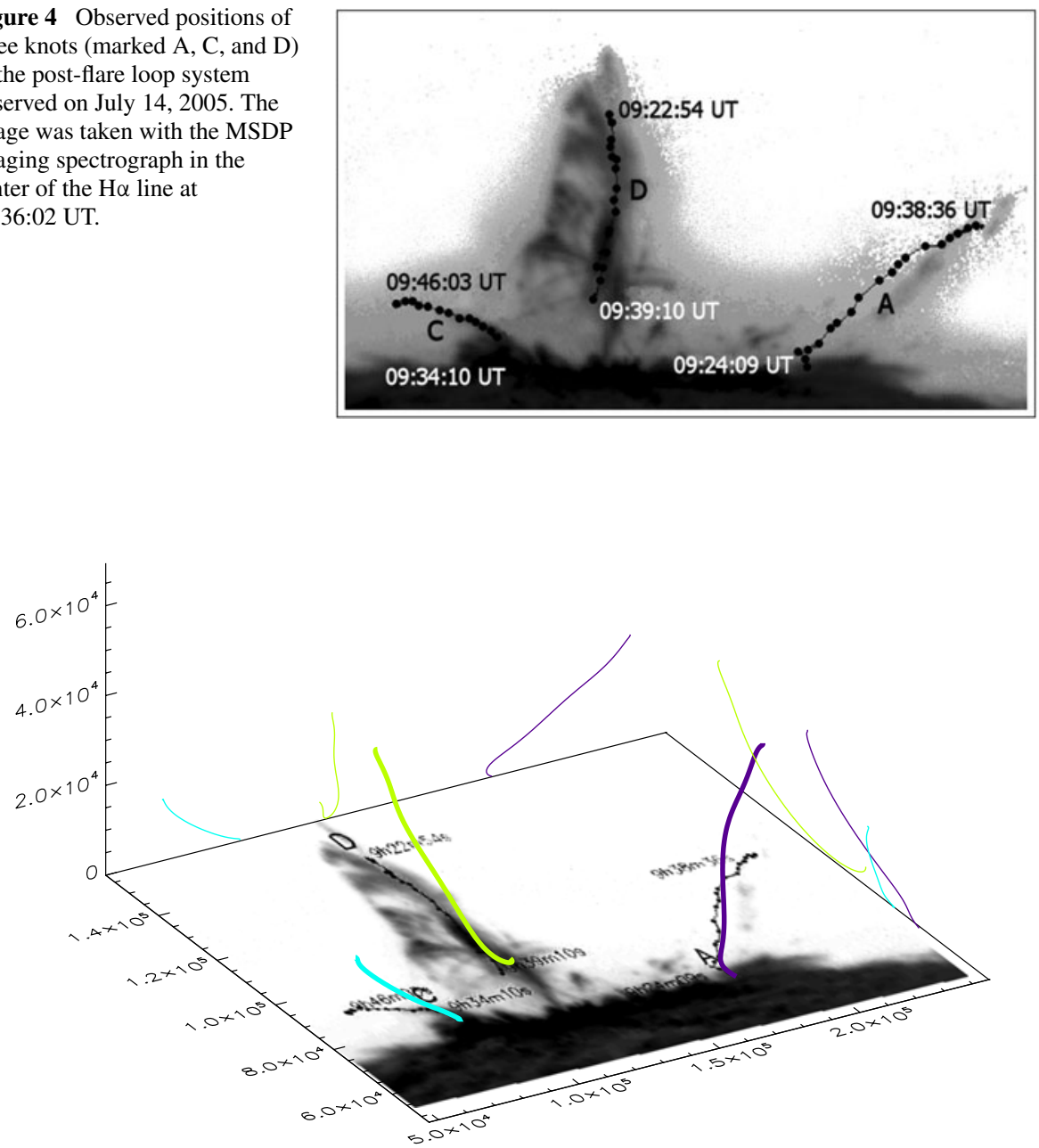

Figure 5 Restored 3D trajectories of the three knots (marked A, C, and D) observed in the post-flare loop system on July 14, 2005. The tracks are presented in the 3D space and overlaid on the image taken with the MSDP spectrograph in the center of the $\mathrm{H} \alpha$ line at 09:36:02 UT. Axes are scaled in kilometers. $Z$ axis (LOS) is vertical. Thick lines of various colors indicate $3 \mathrm{D}$ tracks of the individual knots (knot A - purple, $\mathrm{C}-$ light blue, D - light green, respectively). Projections of the restored 3D track on $X-Z$ and $Y-Z$ planes (both are perpendicular to the plane of the sky), as well as on the plane of the sky $(X-Y)$ are plotted with thin lines. All trajectories are arbitrarily shifted along the $Z$ axis in such a way that the most distant position of each trajectory lies in the plane of the sky $(X-Y)$. 


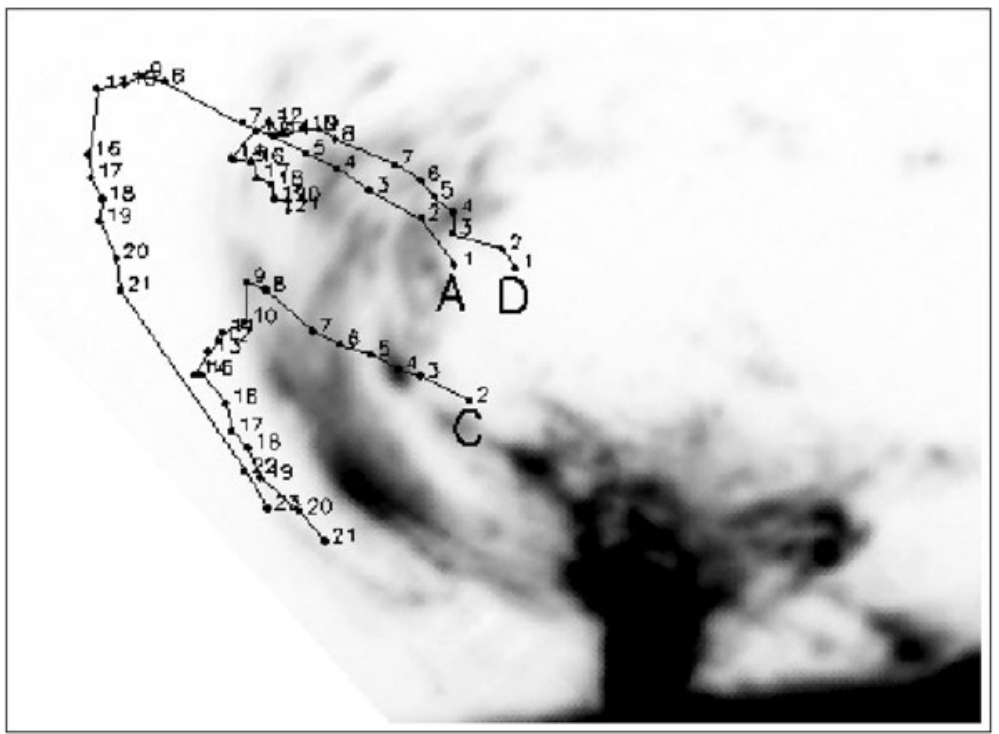

Figure 6 Observed positions of three knots (marked A, C, and D) of the prominence observed on June 1, 2003. The image was taken with the MSDP imaging spectrograph in the center of the H $\alpha$ line at 8:32:42 UT.

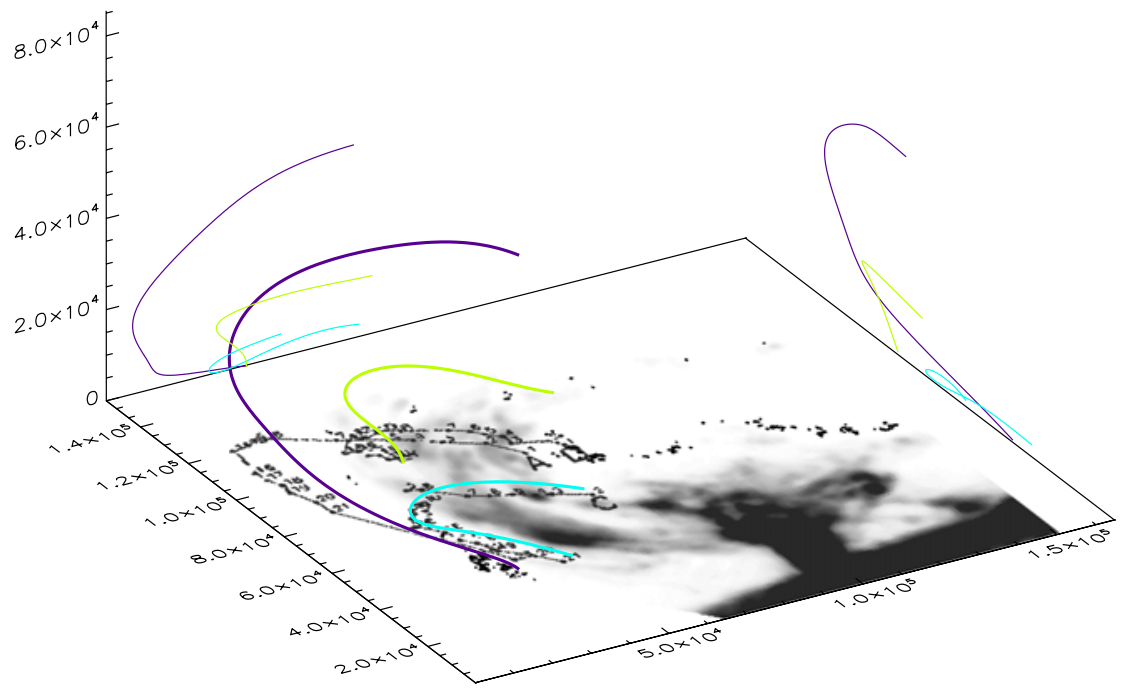

Figure 7 Restored 3D trajectories of the three knots (marked A, C, and D) observed in the prominence on June 1, 2003. See Figure 5 caption for details. An animated version of this figure is available in the electronic version of the paper.

\subsection{Eruptive Prominence on June 1, 2003}

The eruptive prominence was observed from 08:30:12 UT up to 09:13:57 UT near the active region NOAA 10375 on the east solar limb on June 1, 2003. An image of the prominence taken at 8:31:06 UT in the $\mathrm{H} \alpha$ line is presented in Figure 6, where trajectories of the selected 
Table 2 Knots observed in the eruptive prominence on June 1, 2003.

Table 3 Knots observed in the prominence on September 23, 2009.

\begin{tabular}{llll}
\hline Knot & Start obs. & End obs. & No. of images \\
\hline A & $8: 30: 12$ & $8: 56: 12$ & 19 \\
C & $8: 31: 06$ & $8: 51: 40$ & 20 \\
D & $8: 30: 12$ & $8: 51: 40$ & 21 \\
\hline
\end{tabular}

\begin{tabular}{lllll}
\hline Knot & Start obs. & End obs. & No. of images & Mean Doppler vel. \\
\hline 1 & $10: 56: 19$ & $11: 15: 39$ & 45 & $8.4 \mathrm{~km} \mathrm{~s}^{-1}$ \\
2 & $11: 24: 57$ & $11: 45: 11$ & 43 & $2.3 \mathrm{~km} \mathrm{~s}^{-1}$ \\
3 & $13: 58: 26$ & $14: 00: 54$ & 9 & $4.1 \mathrm{~km} \mathrm{~s}^{-1}$ \\
4 & $12: 55: 16$ & $13: 04: 53$ & 26 & $0.6 \mathrm{~km} \mathrm{~s}^{-1}$ \\
5 & $11: 22: 07$ & $11: 30: 45$ & 23 & $15.6 \mathrm{~km} \mathrm{~s}^{-1}$ \\
6 & $12: 34: 44$ & $12: 46: 21$ & 40 & $15.1 \mathrm{~km} \mathrm{~s}^{-1}$ \\
\hline
\end{tabular}

knots projected on the sky plane are also shown. The restored 3D tracks of all knots are presented in Figure 7. Table 2 summarizes the observations of the knots.

At the beginning of the observational period all knots had small radial velocities, but at the end of the observations knots marked $\mathrm{A}$ and $\mathrm{C}$ reached radial velocities of around $40 \mathrm{~km} \mathrm{~s}^{-1}$. The recorded trajectories of the separated knots are combinations of motions of magnetic ropes as a whole and of their own motions within (along) the loops.

\subsection{Quiescent Prominence on September 23, 2009}

A quiescent prominence was observed over the southeast part of the solar limb, close to active region NOAA 11026, on September 23, 2009. We observed the prominence from 08:07 UT up to 14:14 UT and collected 1000 scans over the whole prominence. The time cadence was between 15 and $18 \mathrm{~s}$. Variable weather conditions caused several short gaps in the observations.

We selected six well-separated knots for further analysis (see Table 3 for details). Due to the large amount of data, we applied a code which tracks selected knots on consecutive images. On each image the code finds the minimum radius of the disk with center in the brightest pixel of the knot which has a percentage of pixels with signal larger than $\mathrm{L}$ and lower than $\mathrm{E}$ in its edge (between the blue and green circles on Figure 8). In the second step the code finds the minimum (but larger than or equal to $L$ ) isophote level which lies inside the disk. This isophote is treated as the knot's border. The position of the knot is defined by the mean coordinates of all points inside the isophote weighted by the pixel signal values. The $L$ value is chosen in the range $70-90 \%$ of maximum brightness of the knot on the evaluated image. We found $E \approx 20 \%$ to be suitable for analysis of the well-separated knots, while $E \approx 50 \%$ was better in more crowded fields. Although the automatic procedure works quite well, each restored track was also examined by the observer.

The reconstructed tracks of the selected knots (marked from 1 to 6) are presented in Figure 9. The knots marked 1, 2, 5, and 6 had similar trajectories; they moved away from the observer, upward from the solar surface, and from left to right in the sky plane. Knots 3 and 4 had small Doppler velocities and moved roughly in the sky plane. 
Figure 8 A portion of an image of a prominence, analyzed with an automatic code for tracking knots on consecutive images. Isophotes $70 \%$ and $90 \%$ are marked in orange and red, respectively. Orange squares represent pixels located on the edge of the delimiting disk with signal larger than $L=70 \%$ of maximum brightness of the knot on the evaluated image. In this example the red line inside the blue circle represents the knot's border.
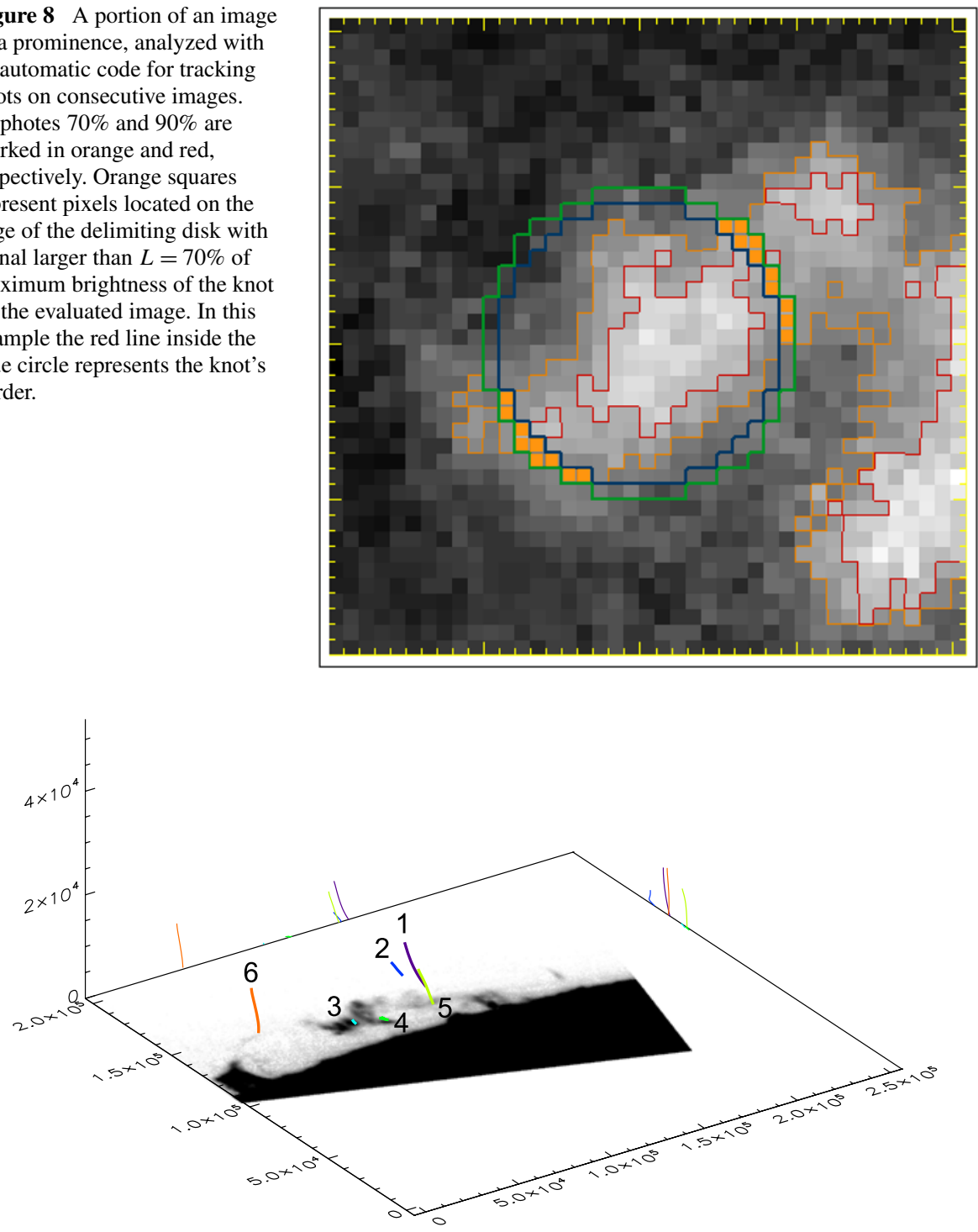

Figure 9 Restored 3D trajectories of the six knots (marked 1-6) observed in the prominence on September 23, 2009. See Figure 5 caption for details. An animated version of this figure is available in the electronic version of the paper.

\section{Discussion and Conclusions}

In this paper we present a new method to restore the true spatial trajectories of prominence knots, based on ground-based observations taken with a single telescope equipped with an MSDP imaging spectrograph. The reconstructed trajectories of several knots observed in three prominences with the Large Coronagraph and MSDP spectrograph installed at Białków Observatory are presented. Our method enables us to evaluate 3D trajectories of the prominence knots without any assumptions about the shapes of the trajectories or the dy- 
namics of the motion. Its only limitation is that the starting positions of the knots along the LOS could not be determined from the data and should be assumed to have any given value (most obviously equal to zero) or should be established from other data. Data like those used in this work, combined with space-based observations, could be very useful for investigating the $3 \mathrm{D}$ evolution and dynamics of prominences, $\mathrm{CME}$ - prominence relations, and the overall evolution of huge magnetic systems.

Open Access This article is distributed under the terms of the Creative Commons Attribution Noncommercial License which permits any noncommercial use, distribution, and reproduction in any medium, provided the original author(s) and source are credited.

\section{References}

Aurass, H., Krüeger, A., Rompolt, B., Garczynska, I., Fomichev, V.V., Chertok, I.M., Ishkov, V.N., Urbarz, H.: 1991, Astron. Nachr. 312, 245.

Ballester, J.L., Kleczek, J.: 1983, Solar Phys. 87, 261.

Bemporad, A.: 2009, Astrophys. J. 701, 298.

Billings, D.E., Pecker, J.C.: 1954, C. R. Acad. Sci. Paris 238, 1690.

Kim, K.-S.: 1990, J. Korean Astron. Soc. 22, 31.

Liewer, P.C., De Jong, E.M., Hall, J.R., Howard, R.A., Thompson, W.T., Culhane, J.L., Bone, L., van DrielGesztelyi, L.: 2009, Solar. Phys. 256, 57.

Makhmudov, M.M., Nikolsky, G.M., Zhugzda, Y.D.: 1980, Solar Phys. 66, 89.

McCabe, M.K., Fisher, R.R.: 1970, Solar Phys. 14, 212.

Mein, P.: 1991a, Astron. Astrophys. 248, 237.

Mein, P.: 1991b, Astron. Astrophys. 248, 669.

Paluš, P.: 1972, Bull. Astron. Inst. Czechoslov. 23, 60.

Panasenco, O., Martin, S.F.: 2007, In: American Geophysical Union, Fall Meeting, abstract \#SH41B-07.

Rompolt, B.: 1975, Rotational motions in fine solar structures. Acta Univ. Wratislaviensis, 252.

Rompolt, B., Mein, P., Mein, N., Rudawy, P., Berlicki, A.: 1993, JOSO Ann. Rep., 87.

Rothschild, K., Pecker, J.-C., Roberts, W.O.: 1955, Astrophys. J. 121, 224.

Rudawy, P.: 1996, JOSO Ann. Rep., 159.

Rudawy, P., Madjarska, M.S.: 1998, In: Webb, D.F., Schmieder, B., Rust, D.M. (eds.) New Perspectives on Solar Prominences, ASP Conference Series 150, 63.

Slater, G.L., Aschwanden, M.J.: 2007, In: American Geophysical Union, Fall Meeting, abstract \#SH32A0771

Tandberg-Hanssen, E.: 1995, The Nature of Solar Prominences, Kluwer Academic, Dordrecht.

Vršnak, B., Ruždjak, V., Rompolt, B., Roša, D., Zlobec, P.: 1993, Solar Phys. 146, 147.

Uddin, W., Gaur, V.P., Pande, M.C.: 1994, Astron. Soc. India Bull. 23, 159. 